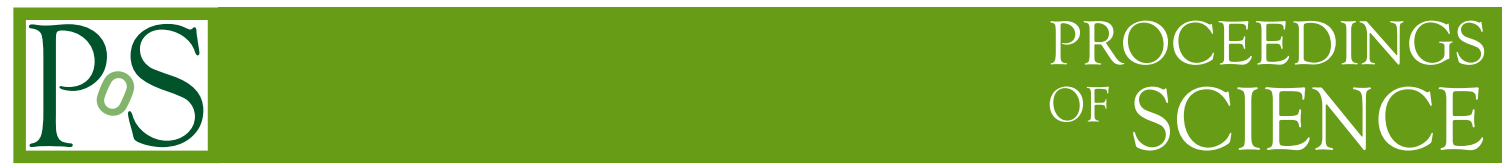

\title{
The Pion Form Factor at Large Momentum Transfer
}

\section{Pai-hsien Jennifer Hsu*}

Yale University

E-mail: pai-hsien.hsulyale.edu

\section{George T. Fleming}

Yale University

E-mail: George.Fleming@Yale.edu

\begin{abstract}
We present our calculations of the electromagnetic form factor of pions. We explore the properties of pion form factor at momentum transfer larger than previous studies by including more combinations of source and sink momenta and using more configurations. We fit our results using vector meson dominance (VMD) hypothesis.
\end{abstract}

The XXV International Symposium on Lattice Field Theory

July 30-4 August 2007

Regensburg, Germany

\footnotetext{
* Speaker.
} 


\section{Motivation}

The pion form factor is often considered a good observable for studying the onset of the perturbative QCD (pQCD)regime in exclusive processes. There are several reasons: First, the asymptotic forms of the pion form factor at both large and small $Q^{2}$ are known. At large $Q^{2}$ it scales as $[1,2,3,4,5,6,7,8,9]$

$$
F_{\pi}\left(Q^{2}\right)=\frac{8 \pi \alpha_{s}\left(Q^{2}\right) f_{\pi}^{2}}{Q^{2}} \quad \text { as } \quad Q^{2} \rightarrow \infty
$$

while at small $Q^{2}$, the pion form factor can be well described by the Vector Meson Dominance (VMD) Model [10, 11, 12]

$$
F_{\pi}\left(Q^{2}\right) \approx \frac{1}{1+Q^{2} / m_{\mathrm{VMD}}^{2}} \text { for } \quad Q^{2} \ll m_{\mathrm{VMD}}^{2}
$$

Therefore at some $Q^{2}$ there must be a transition from the VMD behavior to the large $Q^{2}$ scaling predicted by $\mathrm{pQCD}$. Since the pion is the lightest hadron, the transition is expected to occur at lower $Q^{2}$ than heavier hadrons, which makes it relatively easier to probe by both experiments and Lattice QCD (LQCD). Finally, there is no disconnected diagram on the lattice for the pion form factor. Thus the calculation is pretty straightforward. Previous LQCD studies on the pion form factor can be found in $[13,14,15,16]$ and the references therein.

The current results from various experiments are shown in Fig. 1, including the latest results from Jefferson Lab (JLab) experiments E93-021 [17, 18] and E01-004 [19]. As we can indicate from the figure, the data points around $Q^{2} \sim 2 \mathrm{GeV}^{2}$ start to show some hints of deviation from the VMD fit. This is the energy regime we would like to explore in our study.

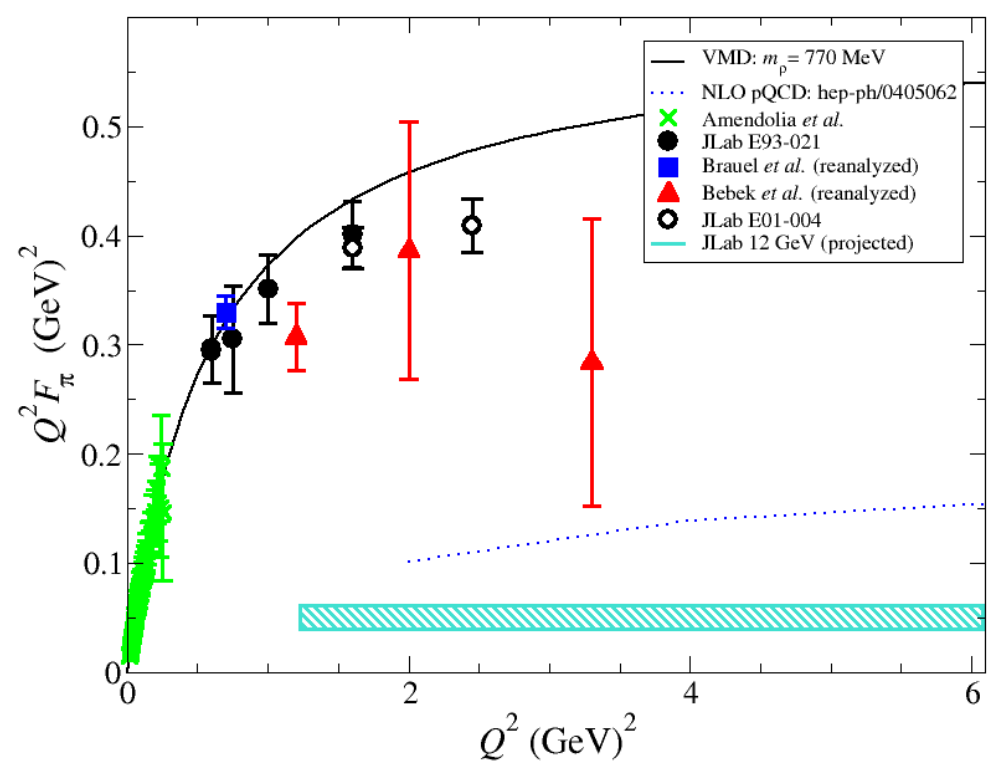

Figure 1: Summary of experimental data for the pion electromagnetic form factor. The two points with open circles are the latest data from the Jefferson Lab (JLab). Shaded regions are expected sensitivities of future experiments. 


\section{Lattice Techniques}

In this section we explain the techniques we used in our lattice calculations, namely the sequential source method (for calculating the quark propagator) and the ratio method (for the correlation functions). The pion electromagnetic form factor is obtained in LQCD by placing a pion creation operator (the "source") at Euclidean time $t_{i}$ with momentum $p_{i}$, a pion annihilation operator (the "sink") at $t_{f}$ with momentum $p_{f}$, and a current insertion at time $t$ with momentum transfer $q$, as shown in Fig. 2. The standard quark propagator calculation provides the two propagator lines that originate from $t_{i}$, the remaining propagator from $t_{f}$ is obtained by the sequential source method: completely specify the quantum numbers and $p_{f}$ at the sink, and contract the propagator from $t_{i}$ to $t_{f}$ with the annihilation operator to serve as the source vector of a second, sequential propagator inversion. The advantage of using the sequential source method is that various currents with different $Q^{2}$ can be inserted at time $t$ without additional matrix inversions. The largest $Q^{2}$ available lies in Breit frame $\left(\vec{p}_{f}=-\vec{p}_{i}\right)$.

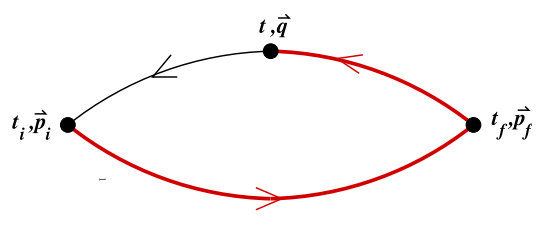

Figure 2: The quark propagators used to compute the pion form factor.

To obtain a simple expression on the lattice, we construct the pion form factor using the ratio method. The pion form factor $F\left(Q^{2}\right)$ is defined as

$$
\begin{aligned}
& \left\langle\pi\left(\vec{p}_{f}\right)\left|V_{\mu}(0)\right| \pi\left(\vec{p}_{i}\right)\right\rangle_{\text {continuum }} \\
& \quad=Z_{V}\left\langle\pi\left(\vec{p}_{f}\right)\left|V_{\mu}(0)\right| \pi\left(\vec{p}_{i}\right)\right\rangle=F\left(Q^{2}\right)\left(p_{i}+p_{f}\right)_{\mu}
\end{aligned}
$$

where $V_{\mu}(x)$ is the chosen vector current. We can extract $F\left(Q^{2}\right)$ from some ratio of the three-point correlation function and the two-point functions. The three-point function can be written as

$$
\begin{aligned}
& \Gamma_{\pi \mu \pi}^{A B}\left(t_{i}, t, t_{f}, \vec{p}_{i}, \vec{p}_{f}\right)=a^{9} \sum_{\vec{x}_{i}, \vec{x}_{f}} e^{-i\left(\vec{x}_{f}-\vec{x}\right) \cdot \vec{p}_{f}} \\
& \quad \times e^{-i\left(\vec{x}-\vec{x}_{i}\right) \cdot \vec{p}_{i}}\left\langle 0\left|\phi_{B}\left(x_{f}\right) V_{\mu}(x) \phi_{A}^{\dagger}\left(x_{i}\right)\right| 0\right\rangle
\end{aligned}
$$


where $\phi$ 's are operators with pion quantum numbers; $A \in(L, S)$ and $B \in(L, S)$ denote either "local" $(L)$ or "smeared" $(S)$. Inserting complete sets of hadron states and requiring $t_{i} \ll t \ll t_{f}$, gives

$$
\begin{aligned}
& \Gamma_{\pi \mu \pi}^{A B}\left(t_{i}, t, t_{f}, \vec{p}_{i}, \vec{p}_{f}\right) \rightarrow\left\langle 0\left|\phi_{B}(x)\right| \pi\left(\vec{p}_{f}\right)\right\rangle \\
& \quad \times\left\langle\pi\left(\vec{p}_{f}\right)\left|V_{\mu}(x)\right| \pi\left(\vec{p}_{i}\right)\right\rangle\left\langle\pi\left(\vec{p}_{i}\right)\left|\phi_{A}^{\dagger}(x)\right| 0\right\rangle \\
& \quad \times \frac{a^{3}}{4 E_{\pi}\left(\vec{p}_{f}\right) E_{\pi}\left(\vec{p}_{i}\right)} e^{-\left(t_{f}-t\right) E_{\pi}\left(\vec{p}_{f}\right)} e^{-\left(t-t_{i}\right) E_{\pi}\left(\vec{p}_{i}\right)} .
\end{aligned}
$$

Similarly for the two-point correlator,

$$
\begin{aligned}
& \Gamma_{\pi \pi}^{A B}\left(t_{i}, t_{f}, \vec{p}\right) \rightarrow\left\langle 0\left|\phi_{B}\left(x_{i}\right)\right| \pi(\vec{p})\right\rangle \\
& \quad \times\left\langle\pi(\vec{p})\left|\phi_{A}^{\dagger}\left(x_{i}\right)\right| 0\right\rangle \frac{a^{3}}{2 E} e^{-\left(t_{f}-t_{i}\right) E} .
\end{aligned}
$$

We can obtain $F\left(Q^{2}\right)$ from the following ratio

$$
\begin{aligned}
F\left(Q^{2}\right)= & \frac{\Gamma_{\pi 4 \pi}^{A B}\left(t_{i}, t, t_{f}, \vec{p}_{i}, \vec{p}_{f}\right) \Gamma_{\pi \pi}^{C L}\left(t_{i}, t, \vec{p}_{f}\right)}{\Gamma_{\pi \pi}^{A L}\left(t_{i}, t, \vec{p}_{i}\right) \Gamma_{\pi \pi}^{C B}\left(t_{i}, t_{f}, \vec{p}_{f}\right)} \\
& \times\left(\frac{2 Z_{V} E_{\pi}\left(\vec{p}_{f}\right)}{E_{\pi}\left(\vec{p}_{i}\right)+E_{\pi} \vec{p}_{f}}\right),
\end{aligned}
$$

where the indices $A, B$ and $C$ can be either $L$ (local) or $S$ (smeared).

\section{Simulation Details and Results}

We use lattices generated by MILC [20], with volume $20^{3} \times 32$ and lattice spacing $a=0.125$ $\mathrm{fm}$. The sea quark mass $m_{\text {sea }}$ and the valence quark mass $m_{v a l}$ are tuned so that we get the same lightest pion mass $m_{\pi}\left(m_{\text {sea }}\right)=m_{\pi}\left(m_{\text {val }}\right)$ [21]. The pion operators are fixed at time $t_{i}=10$ and $t_{f}=20$, and the number of configurations used in this study is 201 . We use five different sets of sink momenta: $\vec{p}_{f}=(0,0,0),(1,0,0),(1,1,0),(1,1,1)$, and $(2,0,0)$.

We present our results in terms of the square of the pion charge radius, obtained by the VMD fit:

$$
\left\langle r_{\pi}^{2}\right\rangle=\frac{6}{m_{V M D}^{2}}
$$

as shown in Fig. 3. The first point on the left is from the data set with only zero sink momentum $\left(\vec{p}_{f}=(0,0,0)\right)$, and for the next point we combined the data from both $\vec{p}_{f}=(0,0,0)$ and $\vec{p}_{f}=$ $(1,0,0)$, and for the third point we added in $\vec{p}_{f}=(1,1,0)$, and so on.

We can see from Fig. 3 that the error bars of $r_{\pi}^{2}$ increases as higher sink momenta are included. Since the pion charge radius is related to the slope of $F\left(Q^{2}\right)$ at low $Q^{2}$, we derive $\left\langle r_{\pi}^{2}\right\rangle$ from the data set of zero sink momentum $\vec{p}_{f}=(0,0,0)$ and $Q^{2}<1 \mathrm{GeV}^{2}$, and check the consistency between the VMD fit and the data above $1 \mathrm{GeV}^{2}$ to see if there is any deviation from the VMD model. 


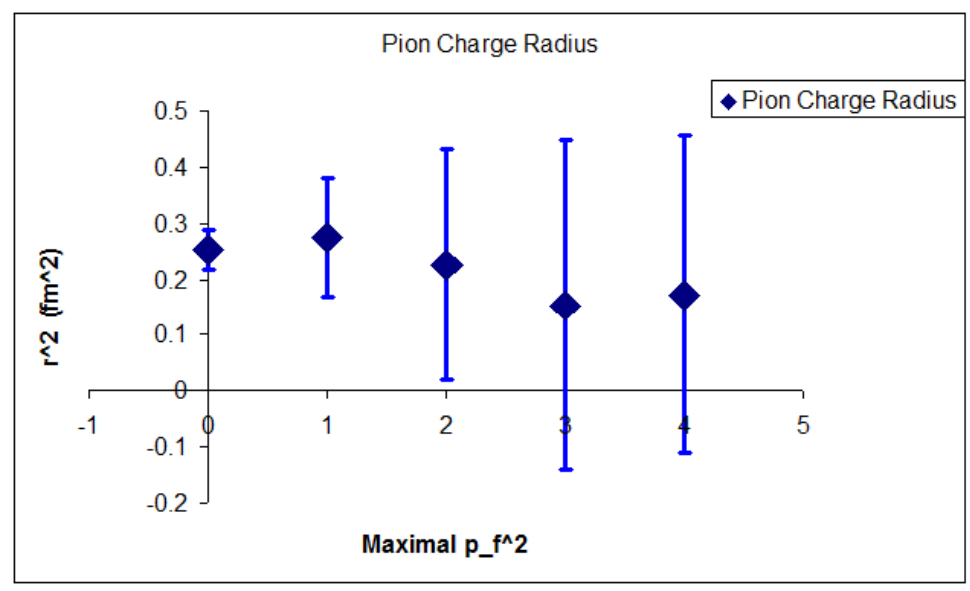

Figure 3: Pion Form Factor VMD fit for $\vec{p}_{f}=(0,0,0)$ to $(2,0,0)$.

The result of this "consistency check" is presented in Fig. 4, where we plot $Q^{2} F\left(Q^{2}\right)$ against $Q^{2}$. While the quantity $Q^{2} F\left(Q^{2}\right)$ should approach a constant as predicted by VMD, we can see that there are some hints of deviation from the VMD model for points with $Q^{2}>2 \mathrm{GeV}^{2}$. To further emphasize this observation, we define $\Delta Q^{2} F\left(Q^{2}\right)=Q^{2} F\left(Q^{2}\right)_{\text {Lattice }}-Q^{2} F\left(Q^{2}\right)_{V M D}$, and plot $\Delta Q^{2} F\left(Q^{2}\right)$ against $Q^{2}$ in Fig. 5 .

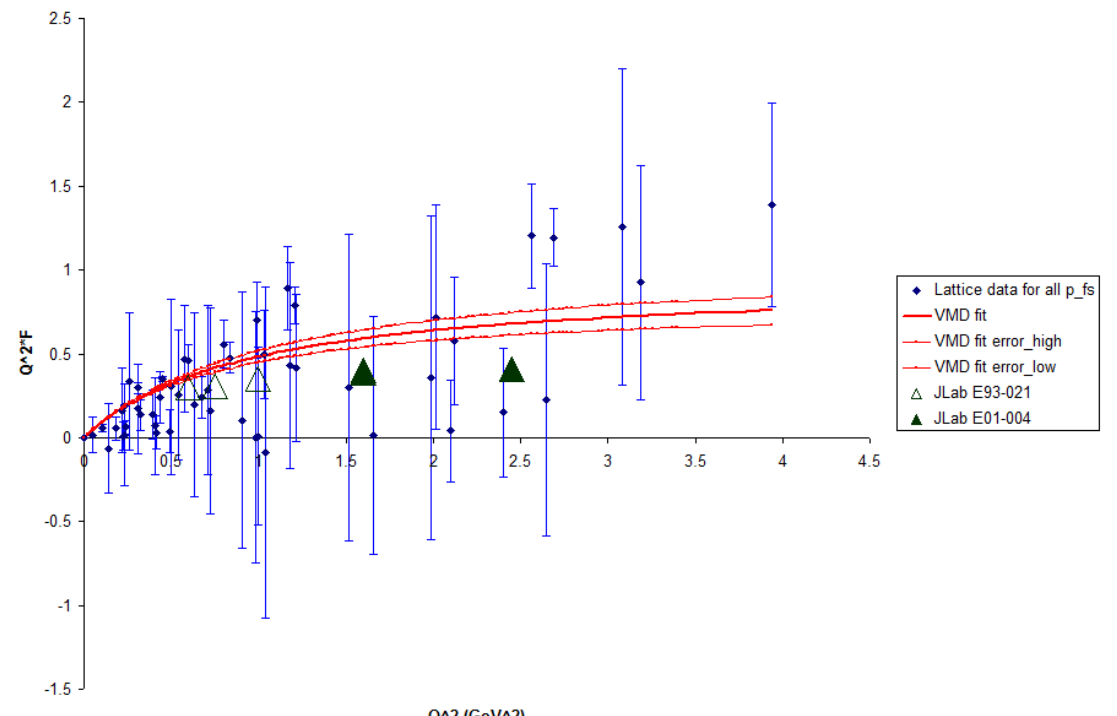

Figure 4: Consistency between data and the VMD fit from low $Q^{2}$ with zero sink momentum. The three red lines represent the VMD fit and its error bars, and the triangle points correspond to the experimental data from JLab.

We also compared this VMD fits from low $Q^{2}$ with that from the Breit frame (where $\vec{p}_{f}=-\vec{p}_{i}$ ), for the data points of the Breit frame have relatively small error bars at high $Q^{2}$. The result is shown in Fig. 6. The figure implies that the VMD fit from the Breit frame (the purple one) is about $1 \sigma$ away from the fit of a single zero sink momentum (the blue one), hence exploring further in the 


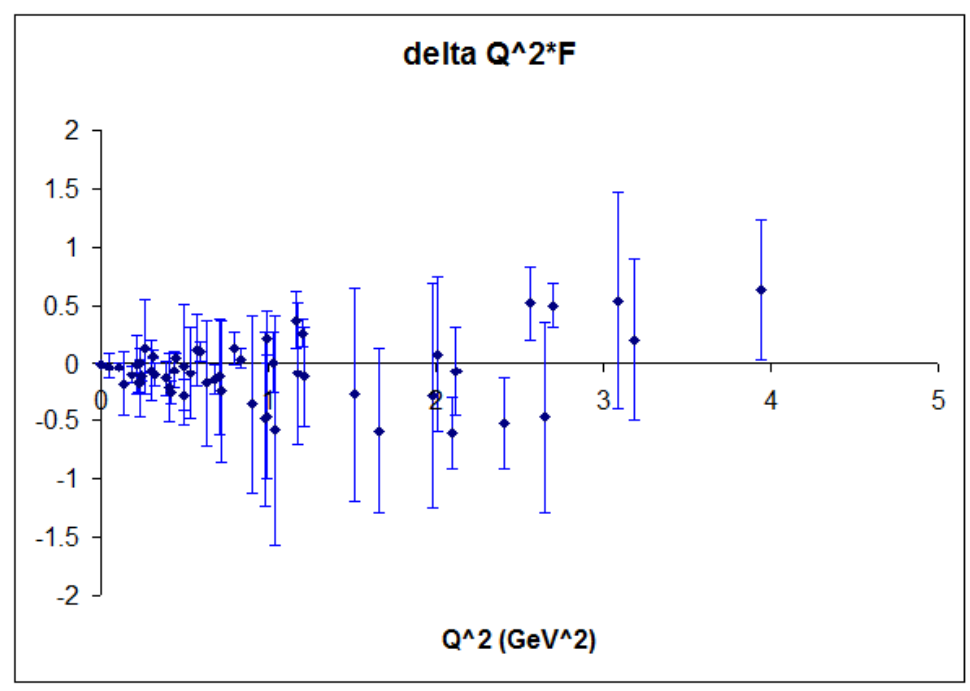

Figure 5: $\Delta Q^{2} F\left(Q^{2}\right)$, as defined in the text.

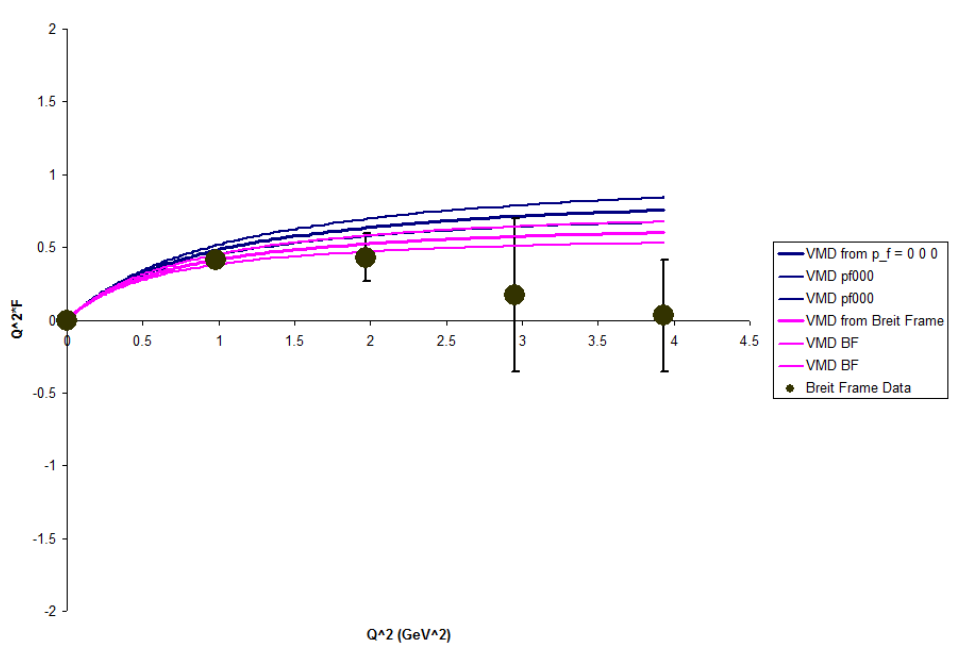

Figure 6: VMD fits with error bands from both data of low $Q^{2}$ with zero $\vec{p}_{f}$, and from data points in the Breit frame.

Breit frame may be the correct direction for studying the pion form factor at higher momentum transfer.

\section{Summary and Outlook}

In this study we have acquired enough lattice data for $Q^{2}<1 \mathrm{GeV}^{2}$ to extract a reliable pion charge radius $r_{\pi}$. By comparing the VMD fit from data with low $Q^{2}$ and high $Q^{2}$, we've started to see some hints of discrepancy between data points at different momentum transfer, which may indicate the transition from the VMD model to pQCD, the goal we are seeking for. We also found that the VMD fit from the Breit frame is about $1 \sigma$ away from the fit of a single zero sink momentum, and we infer that we may explore further in the high $Q^{2}$ regime by studying the data from the Breit 
frame. We are generating four times more data to shrink the error bars in the Breit frame in the hope of a clearer and stronger evidence of the transition into the perturbative QCD regime.

In the meantime, the JLQCD Collaboration has also reported their calculation of the pion form factor based on all-to-all propagators. Interested readers may find details in their upcoming publication [22].

\section{References}

[1] S. J. Brodsky and G. R. Farrar, Phys. Rev. Lett. 31, 1153 (1973).

[2] S. J. Brodsky and G. R. Farrar, Phys. Rev. D 11, 1309 (1975).

[3] G. R. Farrar and D. R. Jackson, Phys. Rev. Lett. 43, 246 (1979).

[4] A. V. Radyushkin, arXiv:hep-ph/0410276.

[5] A. V. Efremov and A. V. Radyushkin,in Proceedings of the XIX International Conference on High Energy Physics, Tokyo, Japan, August 23-30, 1978, edited by S. Homma(Physical Society of Japan,Tokyo,1979), jINR-E2-11535.

[6] A. V. Efremov and A. V. Radyushkin, Theor. Math. Phys. 42, 97 (1980).

[7] A. V. Efremov and A. V. Radyushkin, Phys. Lett. B 94, 245 (1980).

[8] D. R. Jackson, Ph.D. thesis,California Institute of Technology (1977).

[9] G. P. Lepage and S. J. Brodsky, Phys. Lett. B 87, 359 (1979).

[10] W. G. Holladay, Phys. Rev. 101, 1198 (1956).

[11] W. R. Frazer and J. R. Fulco, Phys. Rev. Lett. 2, 365 (1959).

[12] W. R. Frazer and J. R. Fulco, Phys. Rev. 117, 1609 (1960).

[13] F. D. R. Bonnet, R. G. Edwards, G. T. Fleming, R. Lewis and D. G. Richards [Lattice Hadron Physics Collaboration], Phys. Rev. D 72, 054506 (2005) [arXiv:hep-lat/0411028].

[14] S. Hashimoto et al. [JLQCD Collaboration], PoS LAT2005, 336 (2006) [arXiv:hep-lat/0510085].

[15] D. Brömmel et al., PoS LAT2005, 360 (2006) [arXiv:hep-lat/0509133].

[16] D. Brömmel et al. [QCDSF/UKQCD Collaboration], Eur. Phys. J. C 51, 335 (2007) [arXiv:hep-lat/0608021].

[17] J. Volmer et al., Phys. Rev. Lett. 86, 1713 (2001).

[18] V. Tadevosyan et al., Phys. Rev. C 75, 055205 (2007).

[19] T. Horn et al., Phys. Rev. Lett. 97, 192001 (2006).

[20] C. W. Bernard et al., Phys. Rev. D 64, 054506 (2001) [arXiv:hep-lat/0104002].

[21] Ph. Hägler et al. [LHPC Collaboration], arXiv:0705.4295 [hep-lat].

[22] T. Kaneko et al. [JLQCD Collaboration], PoS LAT2007, 148 (2007). 